\title{
Erratum to: Clinical and Histological Features of Nonalcoholic Fatty Liver Disease in Children
}

\author{
Jae Sung Ko $\cdot$ Jung Min Yoon $\cdot$ Hye Ran Yang $\cdot$ \\ Jae Kyung Myung · Haeryoung Kim · Gyeong Hoon Kang • \\ Jung-Eun Cheon $\cdot$ Jeong Kee Seo
}

Published online: 25 September 2009

(C) Springer Science+Business Media, LLC 2009

Erratum to: Dig Dis Sci (2009) 54:2225-2230

DOI 10.1007/s10620-009-0949-3

One of the author names in the published article-Hye Ryeung Kim-is incorrect. The correct name is Haeryoung Kim, as shown in this erratum.

The online version of the original article can be found under doi:10.1007/s10620-009-0949-3.

J. S. Ko · J. M. Yoon · H. R. Yang · J. K. Seo ( $ه)$

Department of Pediatrics, Seoul National University College

of Medicine, 28 Yongondong, Jongrogu, Seoul 110-744, Korea

e-mail: jkseo@snu.ac.kr

J. S. Ko

e-mail: kojs@snu.ac.kr

J. M. Yoon

e-mail: ruleout@empal.com

H. R. Yang

e-mail: hrlamb@hanmail.net

J. K. Myung · H. Kim · G. H. Kang

Department of Pathology, Seoul National University College

of Medicine, 28 Yongondong, Jongrogu, Seoul, Korea

H. Kim

e-mail: medannabel@gmail.com

G. H. Kang

e-mail: ghkang@snu.ac.kr

J.-E. Cheon

Department of Radiology, Seoul National University College

of Medicine, 28 Yongondong, Jongrogu, Seoul, Korea

e-mail: cheonje@snu.ac.kr 